\title{
Linx
}

Revue des linguistes de l'université Paris X Nanterre

$7 \mid 1995$

Saussure aujourd'hui

\section{Analytique de la Phonologie de Saussure : les deux théorèmes}

Jacques Coursil

\section{OpenEdition}

\section{Journals}

Édition électronique

URL : http://journals.openedition.org/linx/1207

DOI : 10.4000/linx.1207

ISSN : 2118-9692

\section{Éditeur}

Presses universitaires de Paris Nanterre

\section{Édition imprimée}

Date de publication : 1 septembre 1995

Pagination : 323-352

ISSN : 0246-8743

\section{Référence électronique}

Jacques Coursil, «Analytique de la Phonologie de Saussure : les deux théorèmes », Linx [En ligne],

7| 1995, mis en ligne le 25 juillet 2012, consulté le 19 avril 2019. URL : http://journals.openedition.org/ linx/1207 ; DOI : 10.4000/linx.1207

Ce document a été généré automatiquement le 19 avril 2019.

Département de Sciences du langage, Université Paris Ouest 


\title{
Analytique de la Phonologie de Saussure : les deux théorèmes
}

\author{
Jacques Coursil
}

Je voudrais seulement ajouter que, vue la nouveauté du problème, .... il serait désirable de présenter vos arguments de la façon la plus claire

et la plus simple sans craindre de les détailler

quelque peu... Mettez-vous à la place d'une

personne n'ayant jamais entendu traiter ces questions . N'oubliez pas que les linguistes, dans leur moyenne, sont des routiniers qui, en outre, sont peu habitués à la matière abstraite...

[Troubetzkoy 1938 XXVI]

1 Le texte des trois conférences sur la Théorie de la syllabe de F. de Saussure [1897] (reproduit dans le Cours de linguistique générale sous le titre Principes de phonologie) se présente aujourd'hui sous le double aspect d'une question d'histoire des sciences non résolue et d'une question technique du plus haut intérêt systémique. Ces deux questions sont liées, l'irrésolution de la première masquant l'intérêt systémique de la seconde. Nous nous contenterons dans cet exposé de situer très succinctement la question d'histoire; ce qui nous permettra d'aborder la question technique plus en détail. Au delà même de sa commodité, cette méthode est épistémologiquement nécessaire car un point d'histoire des sciences ne peut être sérieusement abordé qu'à l'épreuve des faits qui le mettent au jour.

\section{Lecture structuraliste de la phonologie saussurienne}

2 La linguistique s'est fondée comme science, au sens moderne du terme, autour de la découverte du phonème (Baudouin de Courtenay, Saussure) et de la stricte distinction que cette découverte implique entre la phonétique d'une part et la phonologie d'autre part. Mais ce n'est qu'à partir du colloque de Prague [1930], sous l'égide de Troubetzkoy, 
Jakobson, et quelques autres de plus en plus nombreux, notamment Martinet, que la nouvelle méthode s'est véritablement imposée. Entre ce qui est désormais connu sous le nom de Principes de phonologie de Saussure [1897] et le texte pivot de l'Ecole de Prague, Principes de phonologie de Troubetzkoy [1938], on constate à la fois une continuité et une rupture. Les pragois reconnaissent au genevois d'avoir ouvert la voie et installé les études phonologiques sur des bases scientifiquement saines, mais ils lui reprochent d'avoir effectué un revirement contradictoire qui met sa théorie du phonème en amalgame et l'invalide. Cette continuité et cette rupture entre Saussure et l'Ecole de Prague peuvent être montrées au moyen de quelques citations. Dans les Principes de Phonologie, Troubetzkoy, tout en prenant acte de l'héritage saussurien, reproche à Saussure de n'avoir pas appliqué strictement la distinction entre phonétique et phonologie qu'il avait lui-même bâtie. Il écrit :

...Il convient d'instituer non pas une seule mais deux «sciences des sons du langage ", l'une devant avoir pour objet l'acte de parole et l'autre la langue. Leur objet étant différent, ces deux sciences des sons du langage doivent employer des méthodes de travail tout à fait différentes : la science des sons de la parole, ayant affaire à des phénomènes physiques concrets, doit employer les méthodes des sciences naturelles; la science des sons de la langue doit employer des méthodes purement linguistiques, psychologiques ou sociologiques. Nous donnerons à la science des sons de la parole le nom de phonétique et à la science des sons du langage le nom de phonologie. Les linguistes ne sont parvenus que peu à peu à cette distinction entre phonétique et phonologie. (Troubetzkoy 1938 p. 3)

Ferdinand de Saussure, qui a reconnu l'importance de la distinction entre langue et parole et l'a formulée expressément, reconnaît aussi l'essence immatérielle selon son expression du «signifiant » de la langue. Malgré cela, il n'a pas proclamé la nécessité de distinguer une science des sons de la parole » et « une science des sons de la langue »: dans son Cours de Linguistique Générale, cette idée n'est qu'indiquée.

Si F. de Saussure, tout en enseignant que « la langue est un système » n'a pas osé tirer les conséquences logiques de sa propre thèse, cela s'explique en grande partie par le fait qu'une telle conclusion aurait contredit, non seulement la conception usuelle de l'histoire de la langue, mais même les idées courantes sur l'histoire en général (Troubetzkoy 1938 p. XXIV)

Dans son livre intitulé Six leçons sur le son et le sens Jakobson [1941] écrit :

En dépit des nombreuses contradictions dans la doctrine de Saussure, c'est à lui et à son école que nous sommes redevables ...de la notion de rapport entre les phonèmes, bref de la notion de système phonologique. (Jakobson 1941 p. 56)

Des deux aspects du son, c'est donc l'aspect acoustique qui présente avant tout une valeur intersubjective, sociale, tandis que le phénomène moteur, autrement dit le travail de l'appareil vocal, est simplement une condition physiologique du phénomène acoustique. (Jakobson 1941 p. 25)

Ce n'est pas la donnée acoustique en soi qui nous permet de subdiviser la chaîne de la parole en unités distinctes mais seulement la valeur linguistique de cette donnée. La phonétique motrice nous offre une multitude écrasante de variations et il lui manque le critère pour ... nous montrer les invariants à travers la variété..(Jakobson 1941 p. 28-29)

C'est la fonction distinctive, la faculté des sons de différencier les significations des mots... qui importe avant tout.

Les sons munis d'une valeur distinctive, les sons capables de différencier les mots, ont reçu un nom spécial dans la science du langage. On les appelle phonèmes. (Jakobson 1941 p. 44)

Le Cours lance la formule devenue depuis célèbre : les phonèmes sont avant tout des entités oppositives, relatives et négatives. Saussure va jusqu'à affirmer que 
le système phonologique est la seule réalité qui intéresse le linguiste. Mais d'autre part, nous retrouvons dans ce même cours de Saussure l'empreinte d'un psychologisme naïf ... il revient aux procédés de la phonétique motrice.(Jakobson 1941 p. 55)

4 Les pragois reprochent à Saussure de réintroduire dans le débat phonologique des données de phonétique articulatoire, mélangeant ainsi les deux méthodes phonétique et phonologique. En effet, Saussure soutient tout d'abord la stricte distinction des méthodes :

L'essentiel de la langue est étranger au caractère phonique du signe linguistique. [CLG 21]

La langue est une convention, et la nature du signe dont on est convenu est indifférente. La question de l'appareil vocal est donc secondaire dans le problème du langage. [CLG 26]

Il convient de distinguer $: 1^{\circ}$ les combinaisons par lesquelles le sujet parlant utilise le code de la langue en vue d'exprimer sa pensée personnelle; $2^{\circ}$ le mécanisme psychophysique qui lui permet d'extérioriser ces combinaisons (CLG 31)

Considérons, par exemple, la production des sons nécessaires à la parole: les organes vocaux sont aussi extérieurs à la langue que les appareils électriques qui servent à transcrire l'alphabet Morse sont étrangers à cet alphabet; et la phonation, c'est-à-dire l'exécution des images acoustiques, n'affecte en rien le système lui-même. (CLG 36)

Mais, dans le CLG, on peut également lire ce qui suit :

On ne peut réduire la langue au son, ni détacher le son de l'articulation buccale. (CLG 24)

La délimitation des sons de la chaîne parlée ne peut donc reposer que sur l'impression acoustique; mais pour leur description, il en va autrement. Elle ne saurait être faite que sur la base de l'acte articulatoire car les unités prises dans leur propre chaîne sont inanalysables. (CLG 65)

6 Ces citations parmi d'autres laissent apparaitre une contradiction : on ne peut en effet soutenir la nécessité d'une distinction méthodologique entre un champ de données physiques (phonétique articulatoire) et un champ de données symboliques (phonologie), et, en même temps, affirmer que la délimitation des phonèmes dépend de la conformation physiologique de l'appareil vocal.

La liberté de lier les espèces phonologiques (phonèmes) est limitée par la possibilité

de lier les mouvements articulatoires. (CLG 79)

Du strict point de vue de la méthodologie scientifique, d'innombrables travaux, tout au long du siècle, ont montré le bien fondé de l'exigence pragoise selon laquelle les données phonétiques et phonologiques sont ontologiquement et fonctionnellement incompatibles. Ainsi, toutes les preuves d'un revirement contradictoire saussurien semblant réunies, la question est désormais close. En effet, une analyse physicaliste du langage admettant des considérations matérielles dans ses données entre en flagrante contradiction avec le principe d'arbitraire, lequel constitue le premier degré de réalité des études linguistiques et des études symboliques en général. Ce principe indique que les valeurs linguistiques ne sont pas fonction des supports, mais de leurs rapports. Les supports ne contiennent pas les rapports dont ils sont l'objet. Détachés, ils ne contiennent rien du système du langage. Leur analyse au moyen de mesures est linguistiquement non-pertinente. La langue est en dehors de la mesure et du nombre (1938:9) note Troubetzkoy.

Le signifiant est dans la langue quelque chose de tout autre que dans l'acte de parole. (Troubetzkoy 1938 p 9)

La langue est une forme et non une substance (CLG 169) 

sont pas des réalités physiques, mais des immatériaux. Ce sont des valeurs pures émanant strictement du système de la langue. En clair, il faut distinguer le phonème des sons qui le portent. La phonologie, et avec elle la linguistique générale, se fonde sur cette négation et cette mise à l'écart de la phonétique. critique pragoise repose sur un immense malentendu et que, malgré les apparences, la position de Saussure reste fidèle à l'exigence distinctive et n'est pas contradictoire. Pour ce faire, il faut noter tout d'abord qu'entre Saussure et les pragois la continuité méthodologique n'est que relative car, au-delà des concepts fondamentaux partagés, subsiste entre les deux écoles une différence de point de vue, d'objet, de projet et de programme. Le projet de l'Ecole de Prague est un programme structuraliste d'étude du langage ; le projet saussurien est un programme analytique qui s'avère être tout autre chose. Le programme structuraliste partage avec le programme analytique des concepts $\mathrm{du}$ type suivant: arbitraire du signe (premier principe), opposition synchronie/ diachronie, opposition paradigma-tique/syntagmatique et distinction langue/parole. Parmi les concepts saussuriens que la tradition structuraliste n'a pas retenus, on peut noter parmi d'autres, l'opposition nomenclature|inventaire, et la conception de la langue comme structure d'algèbre. Ces différences dans les deux programmes constituent le cadre du malentendu. Les arguments tranchants qui permettent d'affirmer que les « contradictions de Saussure » ne sont que des effets de lecture consistent en ceci : si l'on reprend intégralement et poursuit l'analyse saussurienne, on trouvera à la place des contradictions attendues, deux théorèmes démontrables relevant de la théorie des systèmes. Ces deux théorèmes régissent respectivement les axes paradigmatique et syntagmatique de la grammaire des langues.

10 L'exposé technique qui suit n'est pas une prosopopée. Le programme analytique proposé ici ne sort pas tout armé de Saussure. C'est un programme de quatrième génération reconstruit à la lumière des programmes structuralistes (seconde génération), formalistes (troisième génération) ainsi qu'à l'écoute de la phénoménologie et de la psychanalyse. En outre ce programme saussurien reconstitué est issu du traitement automatique des langues et plus spécifiquement de la systémique et des recherches en Intelligence artificielle. Dans ce cadre, il a été implémenté en plusieurs versions et sur plusieurs langues dans un projet de laboratoire nommé ANADIA ${ }^{1}$.

11 Nous procéderons par une lecture commentée de citations selon une méthode démonstrative, prenant la liberté de remplacer les formalismes souvent lourds employés par Saussure par des notations d'une facture plus moderne, à la fois plus concises et plus lisibles. L'analyse que propose Saussure est restée inachevée. Pour la compléter, il faut parfois, sans changer sa méthode, revenir en arrière, corriger quelques données et affûter quelques hypothèses. En gardant l'oeil sur le texte, le lecteur pourra juger si notre continuation de l'analyse dévie ou non par rapport à la méthode. 


\title{
Le programme analytique de saussurien et la question de la phonologie
}

\section{Argument Withney-Saussure}

Saussure dans un argument célèbre prend position entre ce qu'on peut appeler l'ultraconventionalisme de Whitney [1875] selon lequel l'appareil vocal (APV) n'est pour le langage qu'une commodité instrumentale contingente et le conventionnalisme de Saussure selon lequel cet APV est une fonctionnalité nécessaire.

\begin{abstract}
Ainsi pour Whitney, qui assimile la langue à une institution sociale au même titre que toutes les autres, c'est par hasard, pour de simples raisons de commodité, que nous nous servons de l'appareil vocal comme instrument de la langue : les hommes auraient pu aussi bien choisir le geste et employer des images visuelles au lieu d'images acoustiques. Sans doute cette thèse est trop absolue : la langue n'est pas une institution sociale en tout point semblable aux autres. De plus Whitney va trop loin quand il dit que notre choix est tombé par hasard sur les organes vocaux : ils nous étaient bien en quelque sorte imposés par la nature. Mais sur le point essentiel, le linguiste américain nous semble avoir raison: la langue est une convention. (CLG 26)
\end{abstract}

13 Ce qui ruine la possibilité d'un lien fonctionnel entre phonétique articulatoire et phonologie est que, dans la langue, l'opposition générateur|résonateur n'est jamais pertinente. Ainsi, si Saussure croit pouvoir sans contradiction introduire la nécessité de l'APV dans la fonctionnalité symbolique de la langue, c'est qu'il dispose d'un argument fondamental tranchant. Les deux chapitres "Les espèces phonologiques ", "Le phonème dans la chaîne parlée» constituent la mise en forme et l'exposé de cet argument fondamental.

\section{La catégorie A Apertures (tension|laxité)}

14 A la fois répartie sur les axes paradigmatique et syntagmatique, la phonologie de Saussure surprend par son mode de classement des données. Ce classement qui n'existe pas en phonétique articulatoire et sur lequel la phonologie classique fera l'impasse est crucial pour la fonctionnalité de son système. Il note :

On classe généralement les sons d'après le lieu de leur articulation. Notre point de départ sera différent. Quelle que soit la place de l'articulation, elle présente toujours une certaine aperture, c'est-à-dire un certain degré d'ouverture entre deux limites extrêmes qui sont: l'occlusion complète et l'ouverture maximale. Sur cette base, et en allant de l'aperture minimale à l'aperture maximale, les sons seront classés en sept catégories désignées par les chiffres 0,1,2,3,4,5,6. C'est seulement à l'intérieur de chacune d'elles que nous répartirons les phonèmes en divers types d'après le lieu de leur articulation propre.(CLG 70)

Les catégories d'aperture varient entre deux bornes fixes (entre deux limites extrêmes) :une limite mécanique (occlusion complète), par exemple l'occlusive [p] et une limite symbolique (ouverture maximale), par exemple la voyelle lâche [a] au-delà de laquelle la voix peut aller, mais non pas les langues.

En ce qui concerne la terminologie phonologique, avatar plus qu'héritage de la tradition phonétique qui l'a précédée, Saussure écrit : 
Nous nous conformerons à la terminologie courante, bien qu'elle soit imparfaite ou incorrecte sur plusieurs points : des termes tels que gutturales, palatales, dentales, liquides etc. sont tous plus ou moins illogiques.....(CLG 70)

17 Cette terminologie classique phonético-phonologique a donné lieu a des confusions graves entre la science phonétique et la science phonologique, confusions qui sont aujourd'hui encore courantes. C'est pourquoi, la méthode de Saussure se fonde sur un inventaire chiffré des catégories. Ce recours aux inventaires numériques permet d'éviter certains pièges.

On représente ci-dessous l'inventaire des catégories d'aperture sous forme de tableau. Comme indiqué précédemment, nous n'avons pas hésité à corriger des variables hypothétiques données par Saussure tout en préservant rigoureusement sa méthode.

\begin{tabular}{|c|c|c|}
\hline A & catégories d'aperture & illustrations API \\
\hline 1 & consonnes & $\mathrm{p}$ t $\mathrm{k} \quad \mathrm{b} \quad \mathrm{d} \mathrm{g} \quad \mathrm{f} \int \mathrm{s} \quad \mathrm{v} \quad z_{\mathrm{z}} \mathrm{m} \quad \mathrm{n} \tilde{\mathrm{n}}$ \\
\hline 2 & consonnes liquides & $\mathrm{l} \mathrm{r}$ \\
\hline 3 & semi-consonnes & $\mathrm{W}$ \\
\hline 4 & voyelles tendues & i $y \quad u$ \\
\hline 5 & voyelles moyennes & e $\begin{array}{lllll}\varepsilon & \varepsilon & \varnothing & \partial & \propto\end{array}$ \\
\hline 6 & voyelles lâches & $\tilde{a} \mathrm{a}$ \\
\hline
\end{tabular}

A ce stade de l'analyse, les phonèmes ne sont pas encore définis ; les illustrations API ne sont inscrites dans le tableau qu'à titre indicatif.

On suppose que chaque langue se manifeste par une partition spécifique du champ des apertures qu'elle accepte. Le français, selon Saussure comporte sept rangs d'aperture (qu'une série de tests d'implémentation permet de ramener à six). Ainsi, la partition du champ des apertures constitue un critère primaire de comparaison des langues, deux langues même proches pouvant retenir un nombre différent de rangs d'aperture. On peut illustrer cette classification sous la forme d'un solfège (ou d'un graphe) de tensions d'aperture.

21 [particulièrement] (Schéma des tensions d'aperture)

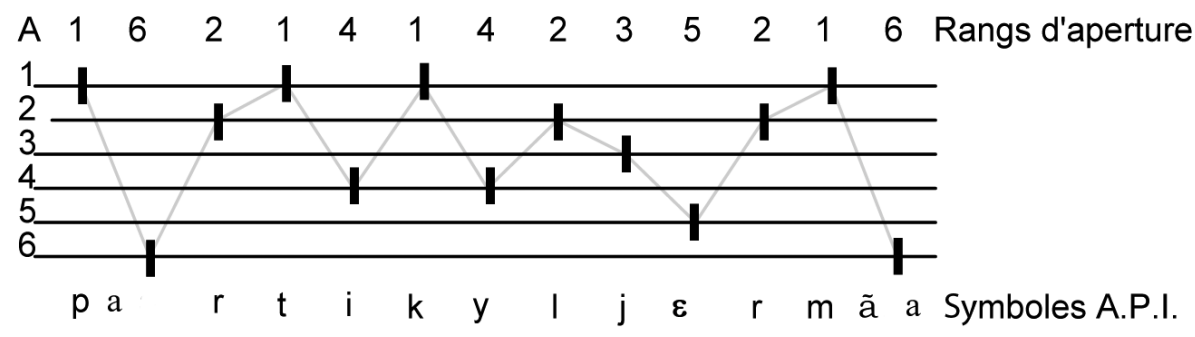

En lisant cette portée comme on pratique le solfège musical, on obtient une notation de l'image acoustique à l'échelle la plus élémentaire. Cette notation indique que la chaîne parlée est articulée par rangs d'aperture.

\section{Axes syntagmatique [P2] et paradigmatique [P1]}

La catégorie des apertures participe fonctionnellement des deux axes syntagmatique (phonématique) [P2] et paradigmatique [P1]. L'axe [P2] délimite les syllabes et l'axe [P1] définit les phonèmes. 


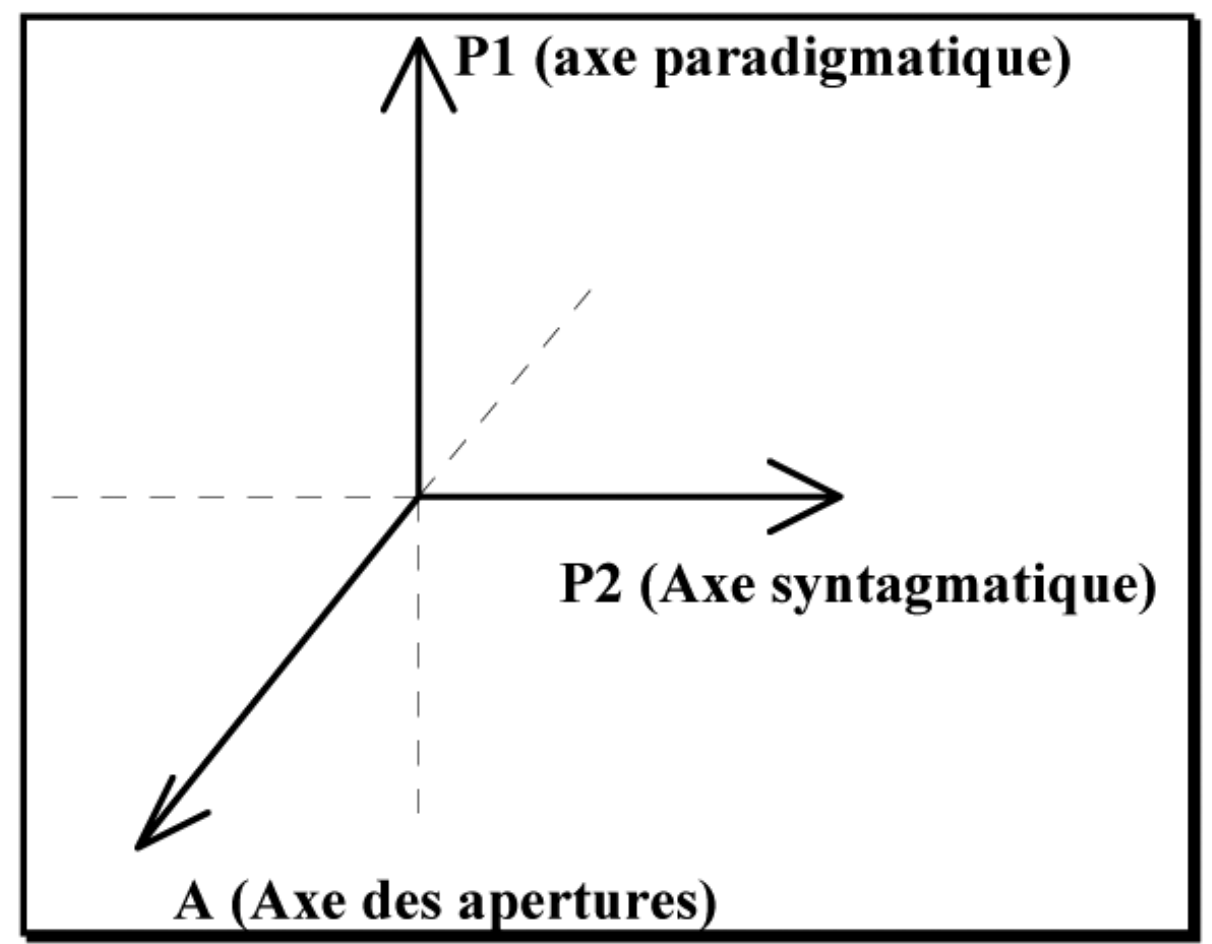

\begin{tabular}{|l|l|l|l|}
\hline$P$ & principes d'effectivité & & \\
\hline 1 & paradigmatique & logique avec identité & phonèmes \\
\hline 2 & syntagmatique & logique sans identité & syllabes \\
\hline
\end{tabular}

Les rapports et les différences entre termes linguistiques se déroulent dans deux sphères distinctes dont chacune est génératrice d'un certain ordre de valeurs. L'opposition entre ces deux ordres fait mieux comprendre la nature de chacun d'eux. Ils correspondent à deux formes de notre activité mentale, toutes deux indispensables à la vie de la langue. (CLG 170)

Ces deux axes P2 et P1 schématisent deux principes d'effectivité différents mais qui prennent pour base un même champ d'apertures [A]. La logique qui régit P2 (théorie de la syllabe) n'admet pas la relation d'identité contrairement à P1(théorie du phonème) qui l'admet. Les deux principes, distincts par leur logique, mais solidaires par le champ A sont structurés algébriquement. Nous commencerons par l'analyse syllabique et terminerons par l'analyse phonologique.

\section{Théorie de la syllabe et théorème P2}

La langue est pour ainsi dire une algèbre qui n'aurait que des termes complexes.

(CLG 168)

Saussure introduit sa théorie de la syllabe par une observation concrète qu'il juge fondamentale. Il écrit :

Nous partons d'une observation fondamentale : quand on prononce un groupe appa, on perçoit une différence entre les deux $p$, dont l'un correspond à une fermeture, le second à une ouverture.

On a appelé la fermeture implosion et l'ouverture explosion; un $p$ est dit implosif (1) ou explosif (0).On peut constater empiriquement cette distinction entre une ouverture (explosion du son) et une fermeture (implosion du son). 


\begin{tabular}{|lll||lll|}
\hline $\mathbf{p}$ & $\mathbf{a}$ & & a & $\mathbf{p}$ & \\
$\mathbf{0}$ & & ouvert & & $\mathbf{1}$ & fermé \\
\hline $\mathbf{b}$ & $\mathbf{a}$ & & a & $\mathbf{b}$ & \\
$\mathbf{0}$ & & ouvert & & $\mathbf{1}$ & fermé \\
\hline $\mathbf{t}$ & $\mathbf{a}$ & & a & $\mathbf{t}$ & \\
$\mathbf{0}$ & & ouvert & & $\mathbf{1}$ & fermé \\
\hline m & $\mathbf{a}$ & & a & $\mathbf{m}$ & \\
$\mathbf{0}$ & & ouvert & & $\mathbf{1}$ & fermé \\
\hline f & $\mathbf{a}$ & & a & $\mathbf{f}$ & \\
$\mathbf{0}$ & & ouvert & & $\mathbf{1}$ & fermé \\
\hline
\end{tabular}

30 L'explosion (ouverture) et l'implosion (fermeture) sont des fonctions de résonance des phonèmes. On les appelle également consonante (fonction 0 ) et sonante (fonction 1 ). Ces fonctions qui n'apparaissent que pour autant que les phonèmes sont disposés en chaîne forment un code booléen qui gère toute la théorie de la syllabe. En principe, tous les phonèmes d'une chaîne sont sujets à l'alternance de ces deux fonctions. Saussure souligne ce point en ces termes:

N'importe quel phonème peut être aussi bien implosif qu'explosif ; mais il est vrai que l'aperture influe sur l'implosion et sur l'explosion, en ce sens que la distinction des deux mouvements devient d'autant moins nette que l'aperture du son est plus grande. (CLG 81)

Deux phonèmes consécutifs se présentent dans la chaîne selon les tables de valeurs suivantes

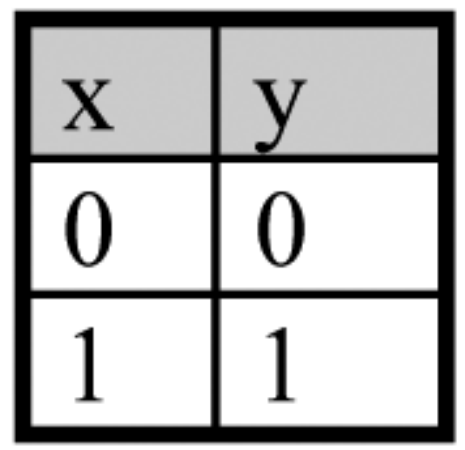

forme développée

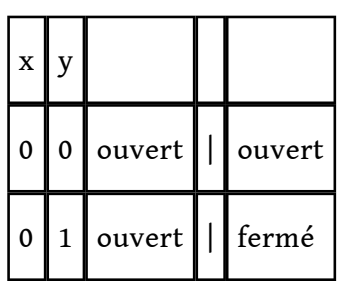




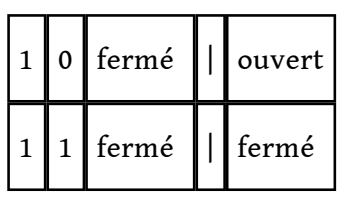


point concret [10] possède une propriété essentielle : c'est l'indice dans la chaîne parlée de la frontière de syllabe.

Si dans une chaîne de sons on passe d'une implosion à une explosion [10], on obtient un effet particulier qui est l'indice de frontière de syllabe. (CLG 86)

La question théorique se réduit donc à celle-ci : si l'on parvient à montrer que dans toute chaîne d'une même langue, le choix des fonctions concrètes pour chaque phonème [0 ou 1] est strictement régi par un code interne au système de la langue, code qui ne prend pas appui sur des mesures acoustiques externes, on aura par là démontré la parfaite cohérence du dispositif méthodologique saussurien. Si en plus, ce système est capable de déconstruire toute suite de phonèmes en une chaîne de syllabes, on reconnaîtra, que les pragois et tous ceux qui les ont suivis dans cette voie, ont fait l'impasse sur ce point important de la linguistique générale.

\section{Code de la langue et phonologie des groupes}

Si dans le phénomène de phonation quelque chose offre un caractère universel qui s'annonce comme supérieur à toutes les diversités locales des phonèmes, c'est sans doute cette mécanique bien réglée...On voit par là l'importance que la phonologie des groupes doit avoir pour la linguistique générale (CLG 79)

Reconstruisons le système de la syllabe. Pour deux phonèmes consécutifs [xy], quatre dispositions de fonctions syllabiques sont possibles :

\begin{tabular}{|l|l|l|l|}
\hline $\mathrm{x}$ & $\mathrm{y}$ & & \\
\hline 0 & 0 & point consonantique & explosion|explosion \\
\hline 0 & 1 & point vocalique & explosion|implosion \\
\hline 1 & 0 & point syllabique & implosion|explosion \\
\hline 1 & 1 & point sonantique & implosion|implosion \\
\hline
\end{tabular}

Les exemples qui suivent sont donnés sans preuves à des fins d'illustration. Les preuves sont données plus loin.

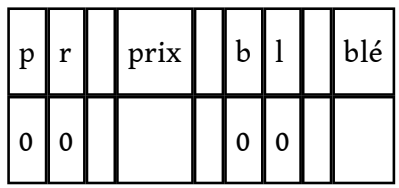

\section{Point vocalique}

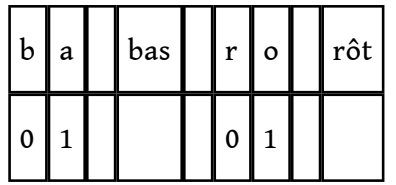




\begin{tabular}{|l|l|l|l|l|l|l|l|}
\hline $\mathrm{k}$ & $\mathrm{S}$ & & action & $\mathrm{r}$ & $\mathrm{m}$ & & particulièrement \\
\hline 1 & 0 & & & 1 & 0 & & \\
\hline
\end{tabular}

\section{Point sonantique}

\begin{tabular}{|l|l|l|l|l|l|l|l|}
\hline $\mathrm{a}$ & 1 & & bal & & $\mathrm{a}$ & $\mathrm{m}$ & femme \\
\hline 1 & 1 & & & 1 & 1 & \\
\hline
\end{tabular}

\section{Conditions d'effectivité du système (règles spécifiques et loi générale)}

\section{Règle des bornes}

47 Toute chaîne complète de phonèmes, autrement dit toute forme syllabique, lexicale ou grammaticale d'une langue, commence par une explosion et se termine par une implosion.

début 0 1 fin

\section{Règle des géminées}

49 Toute consécution de consonnes identiques marque une différence de syllabe [10]

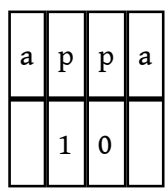

50

\section{Règle des apertures égales}

51 Toute consécution d'apertures égales marque une différence de syllabe [10] (sauf en position finale)

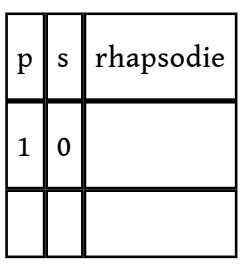

\section{Règle de l'hiatus}

Toute consécution de voyelles marque une différence de syllabe[10] 


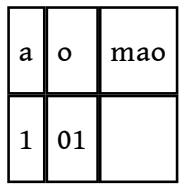
abductivement, c'est-à-dire en commençant par la fin.[0<----1]. Ce renversement de la position positiviste dans l'effectivité des mécanismes est un trait caractéristique de la position analytique propre au programme saussurien, programme qui ne se donne pas pour objet d'expliquer la production phonatoire de la chaîne, mais au contraire donne les règles de sa déconstruction. La démonstration de Saussure est restée inachevée, aussi le théorème P2 n'est pas dans Saussure. Néanmoins, tous les éléments qui concourent à sa mise au point ont été puisés dans les notes du CLG. L'algorithme qui suit s'applique aux cas qui échappent aux règles spécifiques.

\begin{tabular}{|c|c|c|}
\hline A & catégories d'aperture & illustrations API \\
\hline 1 & consonnes & 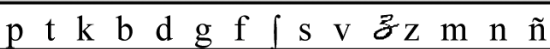 \\
\hline 2 & consonnes liquides & $1 \mathrm{r}$ \\
\hline 3 & semi-consonnes & j $\quad w$ \\
\hline 4 & voyelles tendues & i $\mathrm{y} \mathrm{u}$ \\
\hline 5 & voyelles moyennes & $\mathrm{e} \varepsilon \tilde{\varepsilon} \varnothing$ \\
\hline 6 & voyelles lâches & $\tilde{a} \mathrm{a}$ \\
\hline
\end{tabular}

62 Chaque disposition [10] marque une frontière de syllabe.

63 


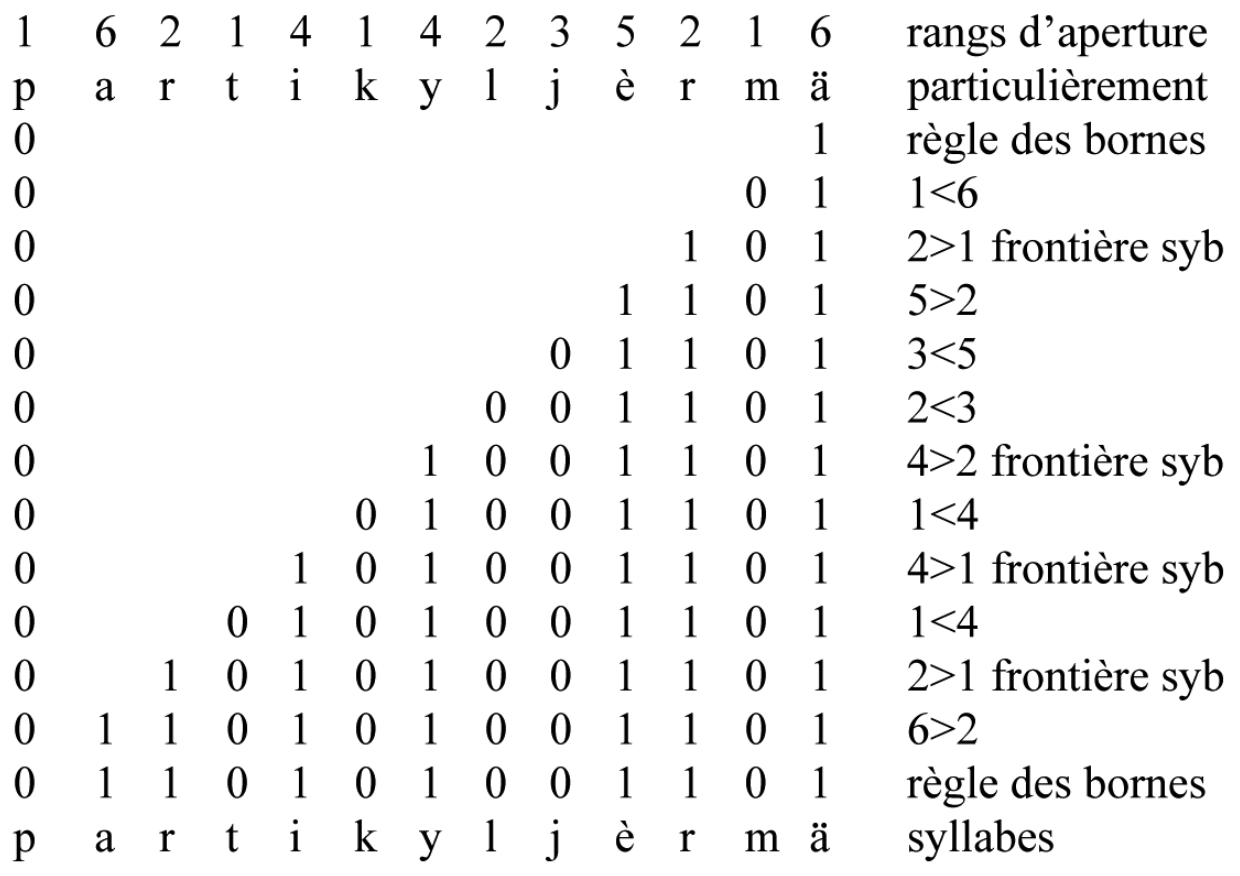

\section{0[par]1 0[ti]1 0[ky]1 0[lje r]1 0[mã ]1}

\section{Cas d'apertures égales}

\begin{tabular}{|l|l|l|l|l|l|l|l|}
\hline 2 & 6 & 1 & 1 & 5 & 1 & 4 & rangs d'aperture \\
\hline $\mathrm{r}$ & $\mathrm{a}$ & $\mathrm{p}$ & $\mathrm{s}$ & $\mathrm{o}$ & $\mathrm{d}$ & $\mathrm{i}$ & rhapsodie \\
\hline $\mathrm{o}$ & 1 & 1 & 0 & 1 & 0 & 1 & syllabes \\
\hline
\end{tabular}

\section{$0[\mathrm{rap}] 10[\mathrm{so}] 10[\mathrm{di}] 1$}

\section{Cas de l'hiatus}

On sait que toute syllabe contient une voyelle. Par ailleurs, les syllabes du français ne contiennent qu'une et une seule voyelle. Ainsi, si une syllabe ne contient qu'un seul phonème, celui-ci est nécessairement une voyelle. Dans la mesure où une syllabe contient toujours les deux types de fonctions 0 et 1 , cette voyelle, traitée comme syllabe, portera aussi les deux fonctions.

\begin{tabular}{|l|l|l|}
\hline V & V & voyelles consécutives \\
\hline 1 & 01 & \\
\hline
\end{tabular}

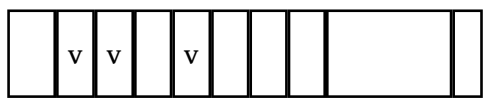




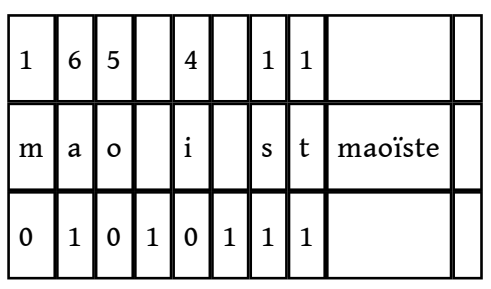

Cas présentent des difficultés liées à leur statut synchro-diachronique. Les cas [st] [ps] notamment sont ambivalents. On les traite au moyen de la règle suivante : les consécutions [st] [ps] (et quelques autres) sont notées [00] quand elles sont en position initiale et [10] dans tous les autres cas.

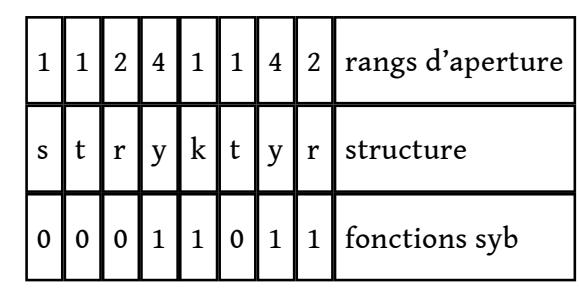

\begin{tabular}{|c|c|c|c|c|c|c|}
\hline 1 & 1 & 4 & 1 & 4 & 1 & rangs d'aperture \\
\hline $\mathrm{s}$ & $\mathrm{t}$ & $\mathrm{y}$ & $\mathrm{p}$ & $\mathrm{i}$ & d & stupide \\
\hline 0 & 0 & 1 & 0 & 1 & 1 & fonctions syb \\
\hline
\end{tabular}

Certains cas restent irréductibles à cette échelle d'analyse notamment [extra], [exquis] [exprès]etc. pour lesquels d'autres règles d'ajustement peuvent être facilement trouvées, mais dont le caractère ad hoc n'ajoute rien au débat en question. Néanmoins des solutions théoriquement plus sérieuses sont formulables. Ces solutions prennent notamment appui sur des règles algorithmiques d'accentuation que nous ne développerons pas ici.

Sur la base de la démonstration qui précède, il semble que la cause selon laquelle la théorie de la syllabe de Saussure ne prend pas appui sur la phonétique est entendue. Le programme saussurien ne met à aucun moment la phonétique et la phonologie en amalgame. Les fonctions syllabiques qu'il propose et les règles qui les régissent sont strictement relatives et différentielles. On a du mal à voir dans le programme de Saussure ce que Jakobson semble y avoir trouvé «l'empreinte d'un psychologisme naif (et un retour) aux procédés de la phonétique motrice ".

Dans la phonologie, les phonèmes n'ont pas de valeur en soi : ce ne sont pas des sons au sens phonétique du terme. Le son dans la phonologie saussurienne commence à la syllabe. Un simple test suffit pour se rendre compte que les phonèmes ne sont pas prononçables isolément. En effet, les consonnes (p,t,k,b,d,g...) sont imprononçables sans une voyelle de soutien avec laquelle elles forment une syllabe. Sans la voyelle, ce ne sont que des intentions manquées dans la bouche. En ce qui concerne les voyelles isolées, nous avons vu qu'elles formaient à elles seules des syllabes portant les deux fonctions d'explosion et d'implosion. $0[\mathrm{i}] 1,0[\mathrm{y}], 0[\mathrm{u}], 0[\mathrm{o}], 0[\mathrm{a}] 1$ etc. C'est donc également comme syllabes et non 
pas comme simples phonèmes qu'elles sont isolément prononçables. Il est symptomatique de constater que la seule voyelle qui fasse exception à cette règle soit précisément le [d] caduc.

La syllabe s'offre plus directement que les sons qui la composent. (CLG 77)

\section{Principe paradigmatique $[\mathrm{P} 1]$ Théorie des phonèmes}

Dans l'analyse syntagmatique (phonématique) de la section précédente, nous n'avons pris appui que sur les fonctions syllabiques et les catégories d'aperture. Nous n'avons mentionné les phonèmes qu'à titre d'illustration sans les définir. En bâtissant la théorie saussurienne des inventaires analytiques, nous allons exposer le système de leur définition. Comme cela a été dit plus haut, la catégorie des apertures qui participe des deux axes P2 et P1 structure l'inventaire paradigmatique. Cet inventaire (pour le français contemporain) contient les sept champs de catégories suivants [ABVNAAO].

\begin{tabular}{|l|l|}
\hline A & apertures \\
\hline B & articulations buccales \\
\hline $\mathrm{V}$ & voisement \\
\hline $\mathrm{N}$ & nasalité \\
\hline $\mathrm{A}$ & arrondies \\
\hline $\mathrm{A}$ & antérieures \\
\hline $\mathrm{O}$ & ouverts \\
\hline
\end{tabular}

Décrivons ces catégories.

\section{Catégorie B dite des articulations buccales}

Appliquons à la catégorie des articulations buccales la même méthode numérique que pour le champ A. Après quelques ajustements, nous obtenons la table suivante :

\begin{tabular}{|c|c|c|}
\hline B & articulations buccales & API \\
\hline 1 & bilabiales & $\mathrm{p} \quad \mathrm{b} \quad \mathrm{m}$ \\
\hline 2 & apicales & $\mathrm{td} \mathrm{n}$ \\
\hline 3 & gutturales & $\begin{array}{lllll}\mathrm{k} & \mathrm{g} & \tilde{\mathrm{n}} & \mathrm{j} & \mathrm{w} \\
\end{array}$ \\
\hline 4 & labiodentales & $\mathrm{f} \mathrm{v}$ \\
\hline 5 & alvéolaires & $\int z$ \\
\hline 6 & post-alvéolaires & $\mathrm{S} \mathrm{Z}$ \\
\hline 7 & latérales-dentales & 1 \\
\hline 8 & latérales-vibrantes & $\mathrm{r}$ \\
\hline 0 & voyelles & $\begin{array}{llllllll}\mathrm{i} \text { y } \mathrm{u} & \mathrm{e} & \varepsilon & \tilde{\varepsilon} \varnothing & \propto & \mathrm{o} & \tilde{\mathrm{a}} \mathrm{a}\end{array}$ \\
\hline
\end{tabular}

La catégorie $B$ des articulations buccales se répartit en huit rangs plus une valeur nulle. En d'autres termes, le champ B est partagé en deux sous-champs notés consonnes dans le 
cas où le rang est supérieur à zéro et voyelles dans le cas où $\mathrm{B}$ est égal à 0 . A ce propos, Saussure note :

La formule d'une voyelle est exactement comparable à celle de n'importe quelle

consonne sonore.

L'opposition consonne|voyelle se définit donc dans l'inventaire au moyen d'une simple formule numérique. On l'écrit [Voyelle ssi B =0].

\section{Voisement, Nasalité, Arrondie, Antériorité, Ouverture}

Les catégories restantes sont binaires. On peut les coder en valeurs booléennes permettant un traitement homogène de l'inventaire numérique.

\begin{tabular}{|l|l|}
\hline $\mathrm{V}$ & Voisement \\
\hline 0 & non-voisée \\
\hline 1 & voisée \\
\hline $\mathrm{N}$ & Nasale \\
\hline 0 & non-nasale \\
\hline 1 & nasale \\
\hline A & Arrondies \\
\hline 0 & non-arrondie \\
\hline 1 & arrondie \\
\hline A & Antérieure \\
\hline 0 & non-antérieure \\
\hline 1 & antérieure \\
\hline $\mathrm{O}$ & Ouvert \\
\hline 0 & non-ouvert \\
\hline 1 & ouvert \\
\hline
\end{tabular}

\section{Registre paradigmatique}

Si nous réunissons les sept champs catégoriques [ABVNAAO] nous obtenons un registre paradigmatique. Le déploiement de ce registre par simple produit cartésien permet, par sélection des cas valides au moyen du critère linguistique de pertinence ${ }^{2}$, de construire l'inventaire analytique de la phonologie d'une langue. Dans cet inventaire, chaque ligne de code représente la description catégorique d'un phonème. Ce registre est minimal. Pour d'autres langues, le registre peut être différent. En créole, par exemple, il ne contient que six catégories. 


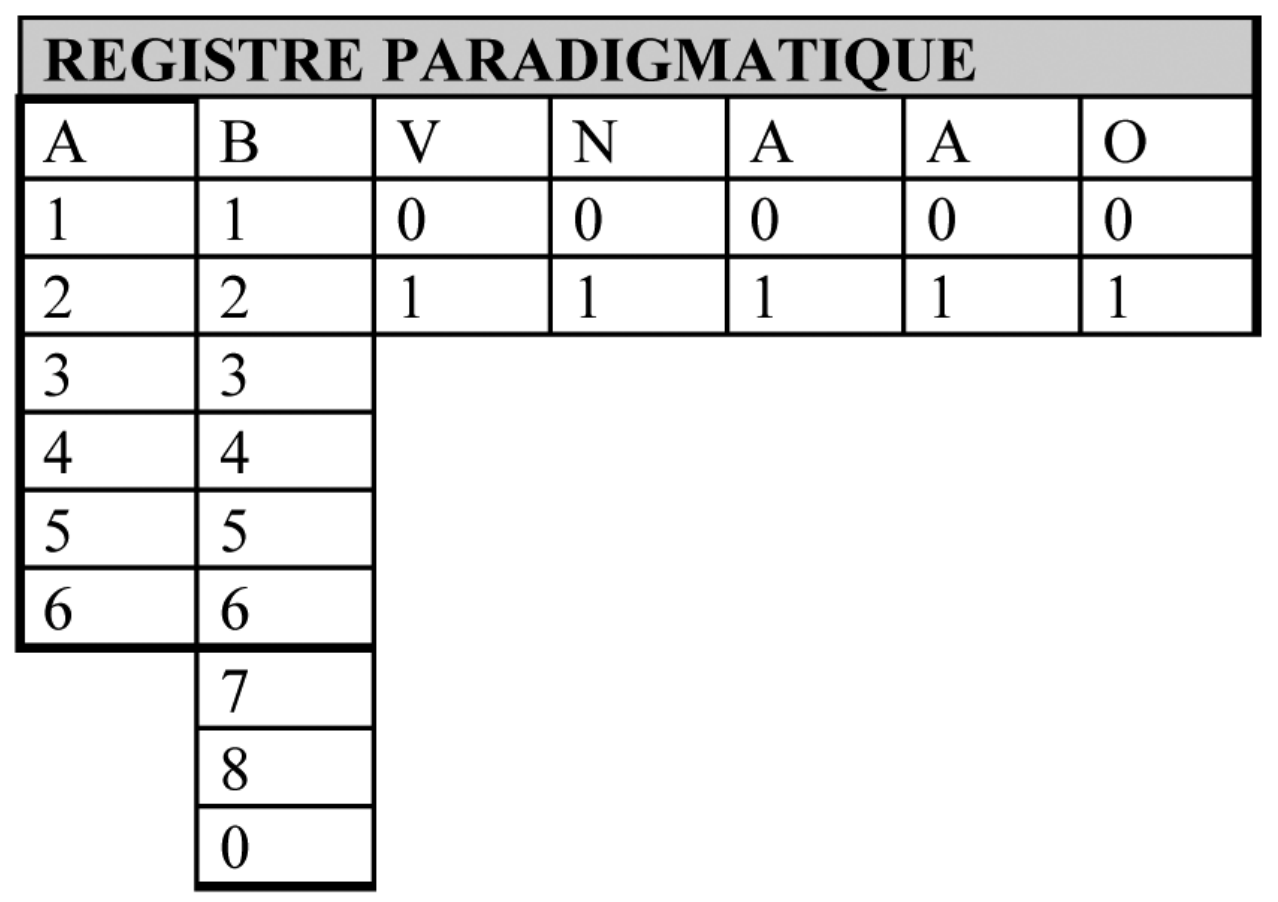

\section{Pertinence, minimalisation et sélection}

Dans le programme analytique saussurien, la phonologie correspond à une échelle d'analyse. Différente en cela des méthodes formalistes qui définissent la phonologie comme une composante satellite d'un corps syntaxique autonome, la phonologie saussurienne correspond à un niveau dans l'architecture générale de la grammaire. On peut simplifier cette architecture en trois échelles $G(1), G(2), G(3)$.

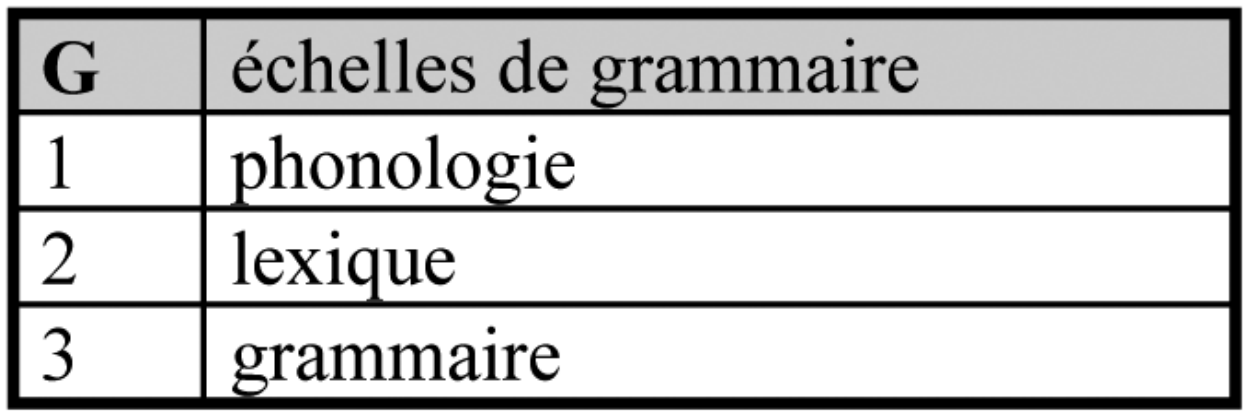

La langue étant un système, il doit y avoir un lien étroit entre la structure grammaticale et la structure phonologique de la langue. (Troubetzkoy XXVII)

De prime abord, cette construction, quoique différente, n'est pas sans rappeler la structuration du langage par Martinet en première et seconde articulations. Néanmoins, si l'on peut y trouver certaines analogies, on y trouve aussi des différences sensibles. Mais en tout état de cause, comme chez Martinet, les échelles de grammaire des langues ne sont pas autonomes, mais solidaires. Elles sont reliées entre elles par un principe de pertinence. Cette pertinence est une fonction qui transite par toutes les échelles de la grammaire. Le principe de pertinence est central dans la théorie du signifiant. Contrairement à une sémantique formelle où la valeur d'un élément est donnée par sa référence selon une procédure de substitution, un élément signifiant ne peut avoir qu'une 
valeur distinctive. De même, un phonème n'est pas une valeur en soi comme le serait un son dans une phonétique : il n'existe dans la langue que pour autant qu'il remplit une fonction distinctive. Les pragois et les fonctionnalistes qui ont mis au point la méthode universellement connue dites des "paires minimales" sont en ce sens les héritiers directs du concept saussurien de pertinence. On emprunte ici leur méthode en la reformulant par une relation plus serrée dite « théorème paradigmatique P1 ».

\section{Minimalisation stricte et théorème paradigmatique $\mathrm{P} 1$}

\section{Exemples}

$\forall \mathbf{x} \in$ phonèmes $\exists \mathbf{y} \in$ phonèmes $\exists$ di $\in$ différence catégorique $(\mathrm{y}+\mathrm{di}=\mathrm{x})$

\begin{tabular}{|l|l|l|l|l|}
\hline $\mathrm{G}$ & & & & \\
\hline 1 & phonologie & b & p & t \\
\hline 2 & lexique & bwa & pwa & twa \\
\hline $\mathbf{3}$ & grammaire & nom & nom & nom \\
\hline
\end{tabular}

\begin{tabular}{|l|l|l|l|l|l|l|l|l|}
\hline A & B & V & N & A & A & O & API & \\
\hline 1 & 1 & 1 & 0 & 0 & 0 & 0 & b & les bois pourrissant \\
\hline 1 & 1 & 0 & 0 & 0 & 0 & 0 & p & les pois pourrissant \\
\hline 1 & 2 & 0 & 0 & 0 & 0 & 0 & t & les toits pourrissant \\
\hline A & B & V & N & A & A & O & API & le pirate ne pensait qu'aux \\
\hline 1 & 1 & 0 & 0 & 0 & 0 & 0 & p & $\begin{array}{l}\text { le pins } \\
\text { butin ne pensait qu'au }\end{array}$ \\
\hline 1 & 1 & 1 & 0 & 0 & 0 & 0 & b & $\begin{array}{l}\text { le pirate ne pensait qu'aux } \\
\text { mutins }\end{array}$ \\
\hline 1 & 1 & 1 & 1 & 0 & 0 & 0 & m \\
\hline
\end{tabular}

Un et un seul rang de catégorie phonologique (1\&1s di) différencie les exemples lexicosémantiques ci-dessus.

La phonologie ne se réduit pas comme la science phonétique à l'étude des sons du langage. Elle se caractérise par le concept de pertinence sémantique et se définit comme l'étude des rapports entre des distinctions acoustiques et des distinctions sémantiques correspondantes. Un trait phonologique suffit pour transformer le sens d'un énoncé et rendre tout un dialogue aberrant. 


\begin{tabular}{|l|l|l|l|l|l|l|l|l|}
\hline A & B & V & N & A & A & O & API & \\
\hline 3 & 3 & 1 & 0 & 0 & 1 & 0 & $\mathrm{j}$ & il aime les miettes \\
\hline 3 & 3 & 1 & 0 & 1 & 1 & 0 & $\mu$ & il aime les muettes \\
\hline 3 & 3 & 1 & 0 & 1 & 0 & 0 & w & il aime les mouettes \\
\hline
\end{tabular}

\begin{tabular}{|l|l|l|l|l|l|l|l|l|}
\hline 5 & 0 & 1 & 0 & 0 & 1 & 1 & $\varepsilon$ & j'ai peur des rats de marais \\
\hline 5 & 0 & 1 & 0 & 0 & 1 & 0 & e & j'ai peur des raz de marée \\
\hline
\end{tabular}

\begin{tabular}{|l|l|l|l|l|l|l|l|l|}
\hline 5 & 0 & 1 & 0 & 0 & 1 & 0 & e & une tablée d'hôtes \\
\hline 5 & 0 & 1 & 0 & 0 & 0 & 0 & $\partial$ & une table d'hôte \\
\hline
\end{tabular}

La pertinence est donc une fonction différentielle à $1 \& 1$ s rang de catégorie près et constitue le principe de base de l'économie signifiante de la langue. En période électorale, on peut imaginer qu'un exemple comme celui qui suit peut jeter le désordre dans la cité :

\begin{tabular}{|l|l|l|l|l|l|l|l|l|}
\hline A & B & V & N & A & A & O & API & \\
\hline 2 & 7 & 1 & 0 & 0 & 0 & 0 & 1 & je vote pour les lois \\
\hline 2 & 8 & 1 & 0 & 0 & 0 & 0 & $\mathrm{r}$ & je vote pour les rois \\
\hline
\end{tabular}

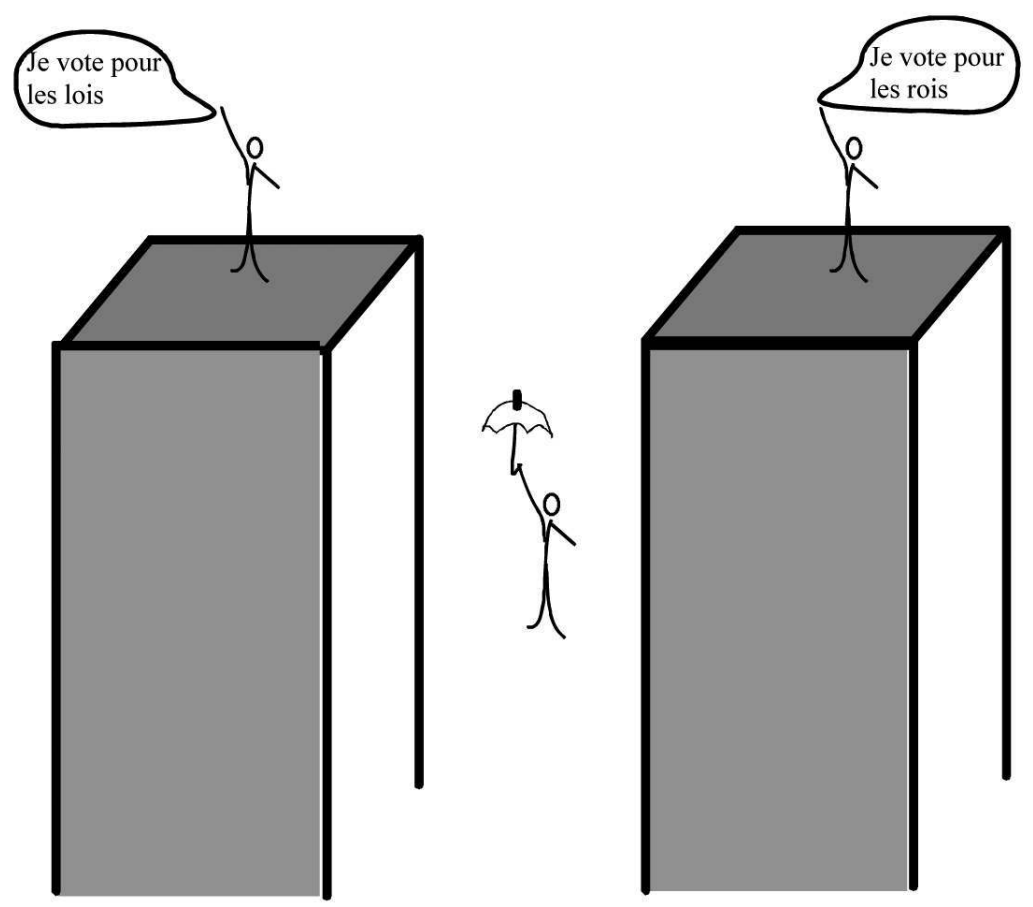

\section{vide signifiant}

Entre les deux catégories (latérale dentale et latérale vibrante) qui distinguent lois de rois, il y a une coupure notée par Saussure élément différentiel. Ce vide signifiant, cette place manquante est précisément celle du sujet. C'est la place d'un savoir divisé dans lequel tout locuteur de la langue se retrouve : entre lois et rois, il n'y a rien (ou bien il y a tout). Cette coupure, élément différentiel, montre à quel point l'analytique saussurienne du signifiant rompt radicalement avec tous les positivismes, que ce soit celui de la phonétique qui considère les sons qu'elle analyse comme des valeurs de langue ou celui de la sémantique classique qui considère les signes comme des objets de sens alors que c'est leur différence qui signifie. Dans l'analytique, l'élément est la marque d'un manque. 
Dans la langue, il n'y a que des différences. Bien plus : une différence suppose en général des termes positifs entre lesquels elle s'établit ; mais dans la langue, il n'y

a que des différences sans termes positifs.(CLG 166)

Le principe constructiviste saussurien est assez proche (et contemporain) de la conception mathématique brouwerienne selon laquelle les mathématiques ne peuvent traiter que d'objets qu'elles ont elles-mêmes construits.

La méthode du programme analytique oppose au concept fixe de nomenclature (classement sous un nom) le concept effectiviste d'inventaire (lat. invenire : découvrir). Cet inventaire n'a pas pour objet de collationner des données comme une nomenclature ; c'est un système qui, à partir d'un jeu de catégories et de fonctions, génère ses valeurs et ses règles dans un certain ordre. Ainsi, comme le souligne Saussure, les valeurs (entités négatives pures) émanent $d u$ système. Entrons dans l'effectivité systémique de cet inventaire.

\section{Inventaire phonologique du français contemporain}

Par application du principe de pertinence sur le registre numérique $\mathrm{P} 1$, on peut construire l'inventaire paradigmatique de la phonologie d'une langue. Dans cet inventaire analytique, les phonèmes, classés selon la méthode saussurienne par rangs d'aperture, sont distincts à un et un seul rang de catégorie près. Ainsi sous un même rang d'aperture, les phonèmes se définissent par un continuum de différences unaires. On énumère l'inventaire agrémenté de quelques exemples.

\begin{tabular}{|c|c|c|c|c|c|c|c|c|c|}
\hline \multicolumn{10}{|c|}{ INVENTAIRE PHONOLOGIQUE DU FRANCAIS } \\
\hline $\mathbf{A}$ & B & $\mathbf{V}$ & $\mathbf{N}$ & $\mathbf{A}$ & $\mathbf{A}$ & $\mathbf{O}$ & API & & \\
\hline 1 & 1 & 0 & 0 & 0 & 0 & 0 & $\mathrm{p}$ & il a avalé une poule & \multirow{15}{*}{ CONSONNES } \\
\hline 1 & 1 & 1 & 0 & 0 & 0 & 0 & $\mathrm{~b}$ & il a avalé une boule & \\
\hline 1 & 1 & 1 & 1 & 0 & 0 & 0 & $\mathrm{~m}$ & il a avalé une moule & \\
\hline 1 & 2 & 1 & 1 & 0 & 0 & 0 & $\mathrm{n}$ & il bat l'ânesse & \\
\hline 1 & 3 & 1 & 1 & 0 & 0 & 0 & $\tilde{\mathrm{n}}$ & il bat la nièce & \\
\hline 1 & 3 & 1 & 0 & 0 & 0 & 0 & $\mathrm{~g}$ & il perd ses gants & \\
\hline 1 & 2 & 1 & 0 & 0 & 0 & 0 & $\mathrm{~d}$ & il perd ses dents & \\
\hline 1 & 2 & 0 & 0 & 0 & 0 & 0 & $\mathrm{t}$ & ce n'est qu'un tri & \\
\hline 1 & 3 & 0 & 0 & 0 & 0 & 0 & $\mathrm{k}$ & ce n'est qu'un cri & \\
\hline 1 & 4 & 0 & 0 & 0 & 0 & 0 & $\mathrm{f}$ & c'est un problème de filage & \\
\hline 1 & 4 & 1 & 0 & 0 & 0 & 0 & $\mathrm{v}$ & c'est un problème de village & \\
\hline 1 & 5 & 1 & 0 & 0 & 0 & 0 & 8 & il vit au milieu des gens & \\
\hline 1 & 5 & 0 & 0 & 0 & 0 & 0 & $\int$ & il vit au milieu des champs & \\
\hline 1 & 6 & 0 & 0 & 0 & 0 & 0 & $\mathrm{~s}$ & un cheval sellé & \\
\hline 1 & 6 & 1 & 0 & 0 & 0 & 0 & $\mathrm{z}$ & un cheval zélé & \\
\hline
\end{tabular}

\begin{tabular}{|l|l|l|l|l|l|l|l|l|l|}
\hline 2 & 7 & 1 & 0 & 0 & 0 & 0 & 1 & il est long & CONSONNES \\
2 & 8 & 1 & 0 & 0 & 0 & 0 & $\mathrm{r}$ & il est rond & LIQUIDES \\
\hline
\end{tabular}

\begin{tabular}{|l|l|l|l|l|l|l|l|l|l|}
\hline & & & & & & & $\mathbf{j}$ & & \\
\cline { 1 - 7 } 3 & 3 & 1 & 0 & 1 & 1 & 0 & & une belle ruée & SEMI- \\
\cline { 1 - 6 } 3 & 3 & 1 & 0 & 1 & 0 & 0 & $\mathrm{w}$ & une belle rouée & CONSONNES \\
\hline
\end{tabular}




\begin{tabular}{|l|l|l|l|l|l|l|l|l|l|}
\hline 4 & 0 & 1 & 0 & 0 & 1 & 0 & $\mathrm{i}$ & un drap de gîte & \\
\cline { 1 - 3 } 4 & 0 & 1 & 0 & 1 & 1 & 0 & $\mathrm{y}$ & un drap de jute & VOYELLES \\
\cline { 1 - 2 } & 0 & 1 & 0 & 1 & 0 & & $\mathrm{u}$ & un drap de joute & TENDUES \\
\hline
\end{tabular}

\begin{tabular}{|c|c|c|c|c|c|c|c|c|c|}
\hline 5 & 0 & 1 & 0 & 0 & 1 & 1 & $\varepsilon$ & la baie est agitée ce soir & \multirow{9}{*}{$\begin{array}{l}\text { VOYELLES } \\
\text { MOYENNES }\end{array}$} \\
\hline 5 & 0 & 1 & 0 & 0 & 1 & 0 & $\mathrm{e}$ & l'abbé est agité ce soir & \\
\hline 5 & 0 & 1 & 0 & 0 & 0 & 0 & & & \\
\hline 5 & 0 & 1 & 1 & 0 & 0 & 0 & $\widetilde{\varepsilon}$ & il a brûlé le pain & \\
\hline 5 & 0 & 1 & 1 & 1 & 0 & 0 & & il a brûlé le pont & \\
\hline 5 & 0 & 1 & 0 & 1 & 0 & 0 & $\mathrm{o}$ & il lui a présenté ses veaux & \\
\hline 5 & 0 & 1 & 0 & 1 & 1 & 0 & $\varnothing$ & il lui a présenté ses voeux & \\
\hline 5 & 0 & 1 & 0 & 1 & 1 & 1 & $œ$ & il a le coeur fatigué & \\
\hline 5 & 0 & 1 & 0 & 1 & 0 & 1 & & il a le corps fatigué & \\
\hline
\end{tabular}

\begin{tabular}{|l|l|l|l|l|l|l|l|l|l|}
\hline 6 & 0 & 1 & 1 & 0 & 0 & 0 & $\tilde{a}$ & c'est un camp militaire & VOYELLES \\
\cline { 1 - 6 } & 0 & 1 & 0 & 0 & 0 & 0 & $a$ & c'est un cas militaire & LACHES \\
\hline
\end{tabular}

La numérisation de l'inventaire et sa disposition par rangs d'aperture n'est pas une variante notationnelle quelconque de la phonologie d'une langue. On a vu comment le champ A permettait de structurer la syllabe au moyen du théorème $\mathrm{P} 2$. On a vu également comment la numération permettait de structurer le paradigme phonologique par une relation serrée définie par le théorème $\mathrm{P} 1$ à $1 \& 1$ s différence près. Mais il y a plus. Contrairement aux structuralistes fonctionnalistes qui considèrent le système de la langue comme une combinatoire ouverte [Martinet 1960], la structure de l'inventaire montre que l'espace phonologique se répartit en quatre composantes connexes, fermées pour la relation proposée. Nous avons appelé topiques ces structures d'algèbre.

\section{Topiques des phonèmes}

101 L'inventaire paradigmatique des phonèmes est réparti par rangs d'aperture Chaque rang est organisé par applications du théorème $\mathrm{P} 1$ qui stipule que pour tout phonème $\mathrm{x}$ il existe au moins un phonème y tel que leur différence soit unaire. Si nous appliquons systématiquement cette condition aux phonèmes classés sous le rang d'aperture $A(1)$, nous obtenons un graphe connexe. Dans ce graphe, chaque ligne reliant deux valeurs représente une différence d'une catégorie et une seule.

Topique des consonnes d'aperture 1 


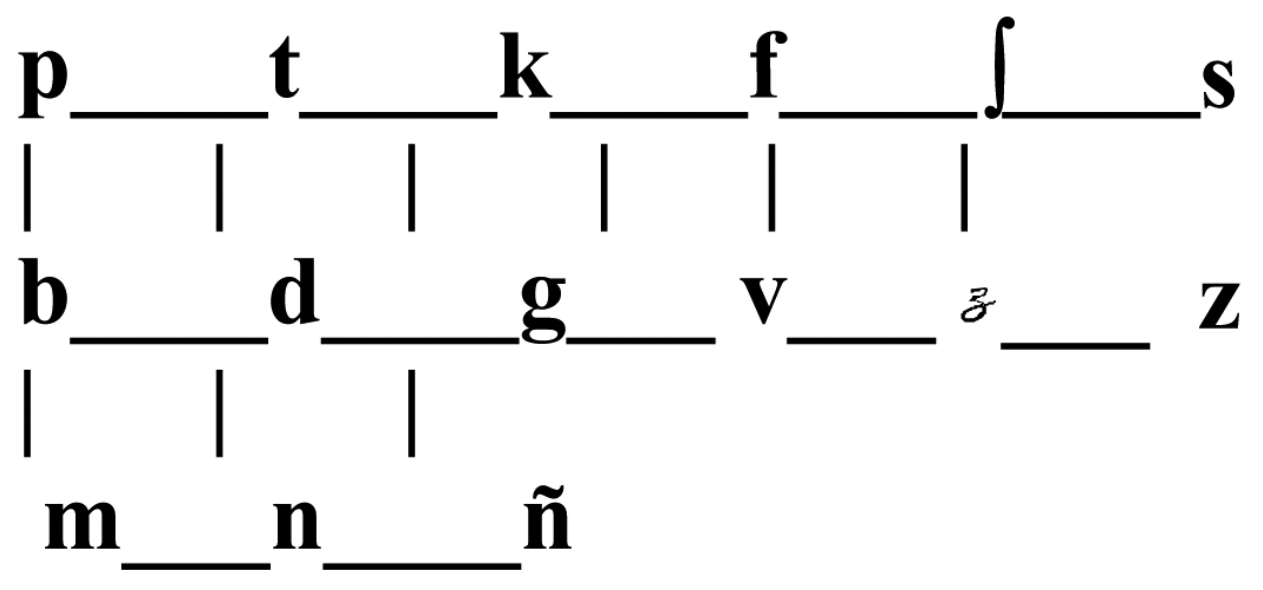

En appliquant strictement le critère de la différence unaire, on constate que les consonnes d'aperture 1 forment, selon l'expression de Saussure, un tout-solidaire où chaque valeur est la transformée d'une autre. On a appelé « topique » cette structure de solidarité fermée parce qu'elle possède des propriétés des espaces connexes. En termes saussuriens, une topique se définit par la solidarité différentielle de ses membres. C'est un système clos, une sorte de jeu de puzzle muni d'une règle du type suivant: soit une valeur $\mathbf{x}$, cherchez l'autre? On peut, en suivant cette règle, effectuer un parcours de différences qui revient récursivement à son point d'origine. Ainsi, le phonème $[\mathrm{g}] \mathrm{a}$ autant de définitions possibles que de possibles parcours différentiels de valeurs.
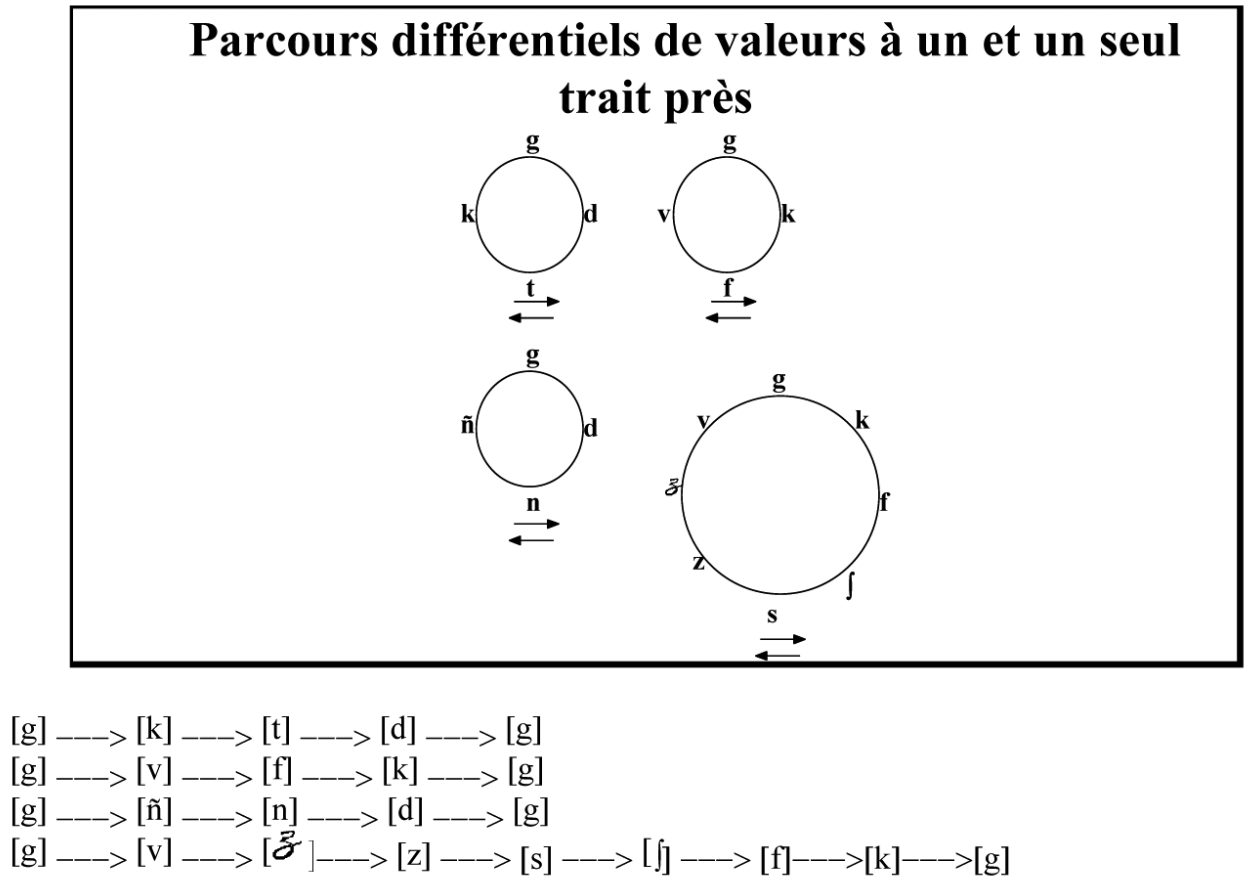

Aperture 2 topique des consonnes liquides

Une analyse semblable peut être effectuée pour les autres rangs d'aperture. 
Aperture 3 topique des semi-consonnes

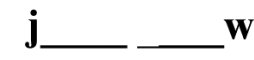

Apertures 4,5,6 topique des voyelles (générée par le logiciel Anadia)

De même que pour les topiques précédentes, chaque ligne reliant deux valeurs représente une différence d'un et un seul trait catégorique.

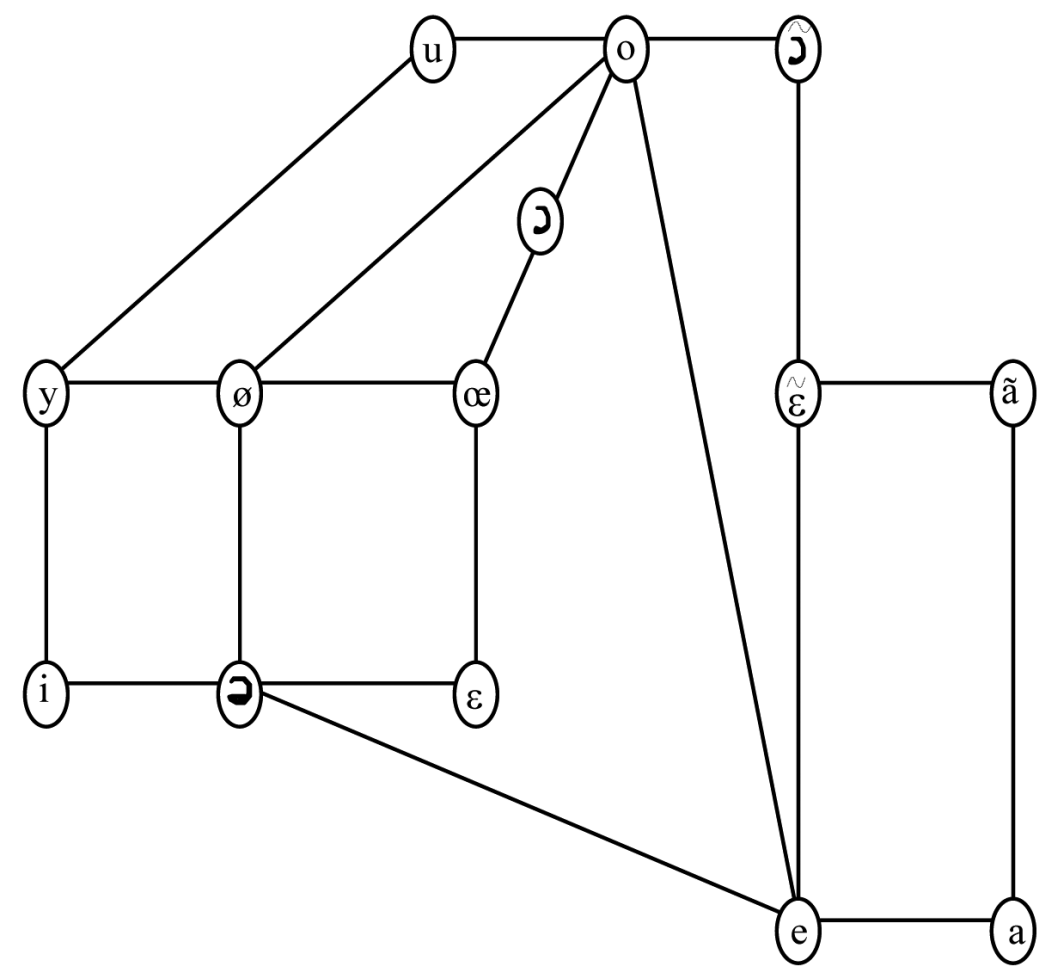

Ainsi, les voyelles forment, elles aussi un système clos, un tout solidaire fermé pour la relation unaire $(1 \& 1 \mathrm{~s} d)$.

107 L'analyse topique souligne sur de nouvelles bases le fait cent fois répété que les phonèmes ne sont pas des entités positives objectives et isolables comme en phonétique, mais des entités négatives, définissables uniquement par différentiation, autrement dit des actes linguistiques de penser ou mieux encore des valeurs pures. Le phonème, comme disent les philosophes, appartient au domaine du pensable. Ainsi, dans le programme saussurien, les termes primitifs ne sont pas des éléments, mais des catégories et des fonctions en termes desquelles le système génère ses valeurs pures. 
La langue présente ce caractère étrange et frappant de ne pas offrir d'entités perceptibles de prime abord, sans qu'on puisse cependant douter qu'elles existent et que c'est leur jeu qui la constitue. (CLG 149) saussurien des valeurs pures, univers resté masqué par la lecture structuraliste de sa théorie. En effet, prévient-il, le point de vue crée l'objet [CLG 23] et les grands linguistes post-saussuriens, tributaires du paradigme métaphysique dans lequel ils étaient inscrits, n'ont pu voir ni l'intérêt de sa démarche syllabique ni celle de sa phonologie. Qu'ils y aient lu des contradictions alors quand y regardant d'un peu près on y découvre des théorèmes reste une énigme que devront résoudre les historiens des sciences du langage. Les générations avancent toujours par bonds note Troubetzkoy[id XXVIII]. Ce bond des pragois, pour être en avant, n'en était pas moins, vis à vis du programme saussurien, un bond de côté. Les épistémologies de nos époques post-modernes nous ont accoutumés au fait que le temps historial n'est pas linéaire et le progrès pas nécessairement droit devant nous. Et, pour ne prendre qu'un exemple, on admet aujourd'hui que la distinction phonétique|phonologie appartient à l'histoire des sciences. Tant de travaux conséquents ont été consacrés à cette question qu'il semble inutile d'y revenir. Et pourtant, après plus de cent ans de débats et de résultats tranchants, elle reste confuse, notamment dans les domaines techniques de la reconnaissance vocale et du traitement automatique des langues. Si le temps historial n'est pas linéaire, il ne semble pas être non plus rétrocirculaire ; je ne crois guère pour ma part à un hypothétique « retour à Saussure » car les retours sont toujours manqués. Il faut savoir se contenter d'une attitude pratique et résultative. Pas de retour à Saussure donc: les textes saussuriens (mis à part les traités) ne sont qu'un agrégat de notes qu'il s'agit de traverser de manière strictement résultative: dans un programme analytique, il ne faut considérer que les évidences analytiques. Je laisse à l'exégèse le soin de l'homme et de l'oeuvre. Je lis dans le texte des éléments d'un programme dont Saussure n'avait peut-être pas l'intention, j'y vois des commodités scientifiques et techniques; peut-être qu'un jour des historiens techniciens attribueront réellement à Saussure, ce que mon analyse en tire littéralement? De toute évidence, cela ne prouvera pas grand chose ni ne rendra la théorie meilleure. Sans me livrer à une archéologie du savoir, j'ai néanmoins tenu à montrer que la démarche analytique de Saussure n'était pas scientifiquement dépassée, loin s'en faut et que la linguistique générale a tout à gagner d'un renouvellement des études saussuriennes. 


\section{NOTES}

1. Les recherches en reconnaisance vocale nécessite la réunion des deux domaines physique et cognitif que sont respectivement la phonétique et la phonologie. La nécessité de cette réunion ne doit pas selon nous, se traduire par un amalgame des données des deux champs, mais par une connaissance fine de leurs différences essentielles.

2. Sur ce concept important de la linguistique générale, voir la contribution de L.PRIETO dans ce même volume. 